\title{
$S$ cerevisiae genome as a confined equilibrium polymer brush
}

\author{
Anton Goloborodko ${ }^{1 *}$, Jon Matthew Belton², Geoffrey Fudenberg ${ }^{3}$, Maxim Imakaev¹, Job Dekker ${ }^{2}$, Leonid Mirny ${ }^{1,4}$ \\ From Epigenetics \& Chromatin: Interactions and processes \\ Boston, MA, USA. 11-13 March 2013
}

\section{Introduction}

A series of recent studies using optical and 3C-based experimental approaches [1,2] have shown that at a global level the yeast genome assumes a Rabl-like conformation with the chromosomal centromeres tethered to the spindle pole body and telomeres anchored on the nuclear periphery. This is strikingly different from human genomic organization, where chromosomes have been shown to assume fractal globule conformations with domains of active and inactive chromatin [3].

In this work, we study Rabl-like chromosomal organization using a computationally efficient polymer lattice model.

\section{Results}

We find that our lattice polymer model predicts experimentally observed $\mathrm{Hi}-\mathrm{C}$ contact maps with high precision $(\mathrm{r} \sim 0.87)$. This demonstrates that a minimal equilibrium model can reconstitute the majority of the observed interaction patterns. Our model predicts that loci preferentially localize to different regions of the nucleus in 3D, depending on their genomic positions. In turn, this leads to different contact preferences between loci. This effect is most clearly demonstrated by the cross-like patterns of interactions between peri-centromeric regions, as observed in yeast $\mathrm{Hi}-\mathrm{C}$ data. Centromere colocalization and excluded volume interactions cause chromatin fibers to extend away from the spindle pole body; this can be understood as a 'polymer brush' effect. In turn, the spatial localization of peri-centromeric loci becomes mainly determined by their genomic distances to the centromere; each locus preferentially forms contacts with other loci at similar distances from their respective centromeres. This dependence of spatial localization on genomic coordinates produces prominent cross-like patterns, as observed in S. cerevisiae Hi-C maps. We estimate this polymer brush effect to alter contact preferences at distances up to $\sim 200 \mathrm{kbp}$ from each centromere. Finally, our lattice model allow us to quickly predict the relative impact of fiber width, flexibility and linear compaction on chromosomal conformation. We find that the observed $\mathrm{Hi}-\mathrm{C}$ maps are consistent with a range of fiber parameters.

\section{Author details}

${ }^{1}$ Department of Physics, Massachusetts Institute of Technology, Cambridge, 02143, MA, USA. ${ }^{2}$ Department of Biochemistry and Molecular Pharmacology, University of Massachusetts, Worcester, 01605, MA, USA. ${ }^{3}$ Graduate Program in Biophysics, Harvard University, Cambridge, 02115, MA, USA. ${ }^{4}$ Division of Health Science and Technology, Harvard-MIT, Cambridge, MA, 02139, USA.

Published: 8 April 2013

\section{References}

1. Duan Z, Andronescu M, Schutz K, Mcllwain S, Kim YJ, Lee C, Shendure J, Fields S, Blau CA, Noble WS: A three-dimensional model of the yeast genome. Nature 2010, 465(7296):363-367.

2. Therizols P, Duong T, Dujon B, Zimmer C, Fabre E: Chromosome arm length and nuclear constraints determine the dynamic relationship of yeast subtelomeres. Proc Natl Acad Sci 2010, 107(5):2025-2030.

3. Lieberman-Aiden E, van Berkum NL, Williams L, Imakaev M, Ragoczy T, Telling A, Amit I, Lajoie BR, Sabo PJ, Dorschner MO, Sandstrom R, Bernstein B, Bender MA, Groudine M, Gnirke A, Stamatoyannopoulos J, Mirny LA, Lander ES, Dekker J: Comprehensive Mapping of Long-Range Interactions Reveals Folding Principles of the Human Genome. Science 2009, 326:289.

doi:10.1186/1756-8935-6-S1-P129

Cite this article as: Goloborodko et al: $S$ cerevisiae genome as a confined equilibrium polymer brush. Epigentics \& Chromatin 2013 6(Suppl 1):P129. 